\title{
Strong-Coupling and Nonlinear Emission from a Quantum-Dot Photonic-Crystal-Slab Nanocavity
}

\author{
J. R. Hendrickson, B. C. Richards, J. Sweet, S. Mosor, G. Khitrova, and H. M. Gibbs \\ Optical Sciences Center, The University of Arizona, Tucson, AZ 85721 \\ jhendrickson@optics.arizona.edu; 302-354-2591; 520-621-4323(fax)
}

T. Yoshie and A. Scherer

Electrical Engineering, California Institute of Technology, Pasadena, CA 91125

yoshie@alumni.caltech.edu; etcher@caltech.edu

\section{O. B. Shchekin and D. G. Deppe}

Microelectronics Research Center, Department of Electrical and Computer Engineering

The University of Texas at Austin, Austin, TX 78712

deppe@ece.utexas.edu

\begin{abstract}
An InAs quantum dot in a photonic crystal nanocavity exhibits vacuum Rabi splitting (strong coupling); a clear anti-crossing is seen between the quantum dot transition and the nanocavity mode as the temperature is scanned.

(C2005 Optical Society of America

OCIS codes: (270.5580) Quantum electrodynamics; (130.3120) Integrated optics devices
\end{abstract}

We will report the pump dependence of the emission of high-Q photonic crystal nanocavities with a few weakly coupled quantum dots within the mode volume and compare it with the emission of a high-Q nanocavity strongly coupled to a single quantum dot.

Our photonic crystal nanocavity follows the design [1] of Noda's group, but their silicon is replaced by GaAs for growth of quantum dots. The three-dimensional-mode in-plane confinement is obtained by fabricating a twodimensional triangular lattice photonic crystal slab with three holes missing to form a spacer. The vertical confinement, achieved by total internal reflection at the slab semiconductor-air interfaces, is imperfect in that light with small in-plane wavevectors can leak out of the top and bottom. Noda's one-dimensional model showed that the key to reducing this loss is to shift out slightly the holes at the ends of the spacer. When the field envelope function is stopped abruptly, its Fourier transform has a larger overlap with small in-plane wavevectors that leak out; terminating it gently reduces that loss.

The sample, grown on a (001) GaAs substrate by molecular beam epitaxy, has a single layer of InAs quantum dots in the center of the slab [2]. The typical dot size is $\sim 25 \mathrm{~nm}$ in diameter and 3-4 nm high, and the dot density is $300-400 / \mu \mathrm{m}^{2}$. A large array of nanocavities $\left(-30,000\right.$ in clusters of 30 with a density of 5,560 cavities $\left.\mathrm{mm}^{-2}\right)$ is fabricated with crystal parameters changed systematically. The missing-holes spacer is surrounded by 14 periods of air holes for good in-plane optical confinement. The parameters of the photonic crystal are controlled lithographically [2]: separation between holes $a=300 \mathrm{~nm}$, hole radius $r=0.27 a$, shift of the two end holes $s=$ $0.20 a$, and slab thickness $d=0.90 a$; see Fig. 1 .

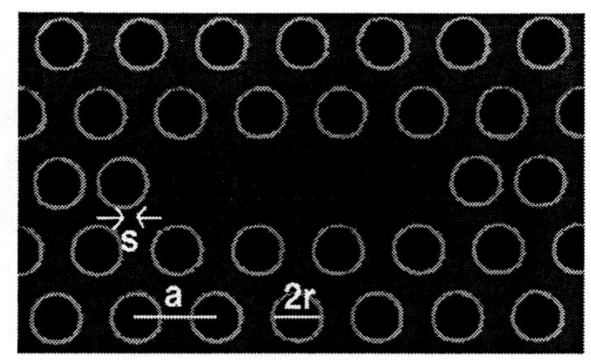

Fig. 1 Scanning electron micrograph of a fabricated photonic-crystal-slab nanocavity showing the hole spacing a, hole diameter $2 r$, and shift $s$ of the two holes at the ends of the "spacer" formed by omitting three holes. 


\section{QME3}

We have observed vacuum Rabi splitting in such a nanocavity between an InAs single quantum dot and a mode of the vacuum field [3]. For that first observation, the excitation was cw at $770 \mathrm{~nm}$. At high pump power $(690 \mu \mathrm{W})$, the many weakly coupled quantum dots were saturated, so that by far the strongest emission occurred at the cavity mode due to enhanced spontaneous emission; see Fig. 2(a). At intermediate pump power (25 $\mu \mathrm{W}$, Fig. 2(b)) the thermal shift to longer wavelength in Fig. 2(a) disappeared, and some photoluminescence peaks from individual single quantum dots began to appear. Figs. 2(c) and 1(d) display the photoluminescence spectra and anti-crossing curve for low pump power as the temperature was scanned to tune the resonance of a strongly coupled single quantum dot through the cavity mode peak of the nanocavity. Plot of an analytic expression for zero-detuning emission using $2 \mathrm{~g}=41.2 \mathrm{GHz}(0.192 \mathrm{~nm})$ for the minimum splitting, $\kappa=42.3 \mathrm{GHz}(0.197 \mathrm{~nm})$ for the cavity decay rate, and $\gamma=21.5 \mathrm{GHz}(0.1 \mathrm{~nm})$ for the dephasing rate of the dot transition agrees well with the data; the condition for strong coupling, $2 \mathrm{~g}>(\kappa+\gamma) / 2$, is satisfied. We have seen anti-crossings using five other nanocavities on a newly fabricated sample with minimum splittings ranging from 0.08 to $0.12 \mathrm{~nm}$.
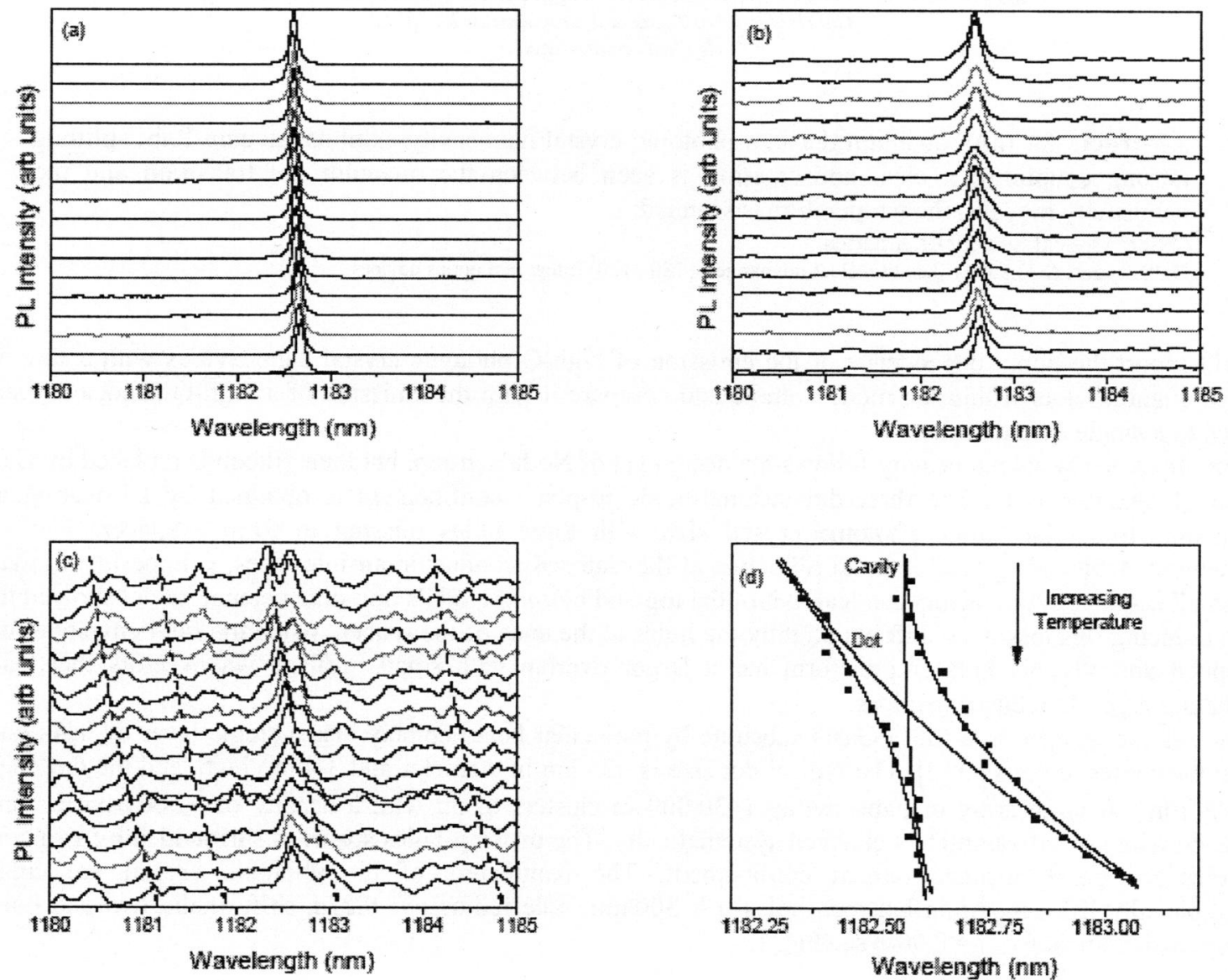

Fig. 2 Dot-nanocavity anti-crossing. In all four figures temperature is scanned from $13 \mathrm{~K}$ at the top to $29 \mathrm{~K}$ at the bottom, in $1 \mathrm{~K}$ steps. (a)-(c) Nanocavity photoluminescence as a function of temperature and excitation power. (a) High power (690 $\mu \mathrm{W})$ and $0.2 \mathrm{~s}$ average time; $\mathrm{Q} \cong 13,300$. (b) Intermediate power $(25 \mu \mathrm{W})$ and $1 \mathrm{~s} ; \mathrm{Q} \cong 8000$. (c) Low power $(0.78 \mu \mathrm{W})$ and $60 \mathrm{~s}$. Note the anti-crossing of one QD transition with the cavity mode at $1182.6 \mathrm{~nm}$. (d) The two coupled-system peaks are plotted as a function of temperature and compared with the scan rates of an uncoupled QD [red curve; from dashed curves in (c)] and an empty cavity [blue curve; from (b)]. The black lines are eye guides to the dot-nanocavity anti-crossing.

Our observation of semiconductor strong coupling followed shortly after the observation of strong coupling between a much larger $(30 \mathrm{~nm}$ by $100 \mathrm{~nm}$ ) InGaAs single quantum dot and the mode of a micropillar cavity with diameter of $1.5 \mu \mathrm{m}$ (mode volume $\sim 0.3 \mu \mathrm{m}^{3}$ compared with our $0.04 \mu \mathrm{m}^{3}$ ) [4]. The smaller volume of the dot here gives larger quantization splittings between levels; this could turn out to be important in future quantum 


\section{QME3}

experiments, such as climbing the Jaynes-Cummings ladder. Since $\mathrm{g}=\mu \mathrm{E}_{\mathrm{vac}} / \mathrm{h}$ (where $\mu$, the dipole moment of the quantum dot, increases with the volume of the dot, and $E_{\mathrm{vac}}$ is the vacuum field satisfying $\left.n^{2} \varepsilon_{0}\left|E_{\mathrm{vac}}\right|^{2} V=h v / 2\right)$, the smaller dot is made possible by the smaller volume $V$ of a photonic crystal nanocavity, $\sim\left(\lambda_{0} / n\right)^{3}$, or about eight times larger than the $\sim\left(\lambda_{0} / 2 n\right)^{3}$ minimum volume. These two breakthroughs signal the beginning of strong coupling studies in semiconductors which have one important advantage over their atomic physics counterparts: the quantum dot does not move or escape, so the same quantum oscillator can be used over and over again. An additional advantage is the integrated structure; everything is locked in place in a very tiny volume.

Experiments now in progress on the nonlinear emission of various nanocavities as a function of pump power will also be reported. The quantum dot nanocavities are pumped with the output of a Tsunami fs Ti:Sa laser gated to a repetition rate of $2 \mathrm{MHz}$ to reduce heating of the membrane nanocavity. The wavelength is $770 \mathrm{~nm}$, and the pulse length after propagation through a $45 \mathrm{~m}$ optical fiber is $>20 \mathrm{ps}$. The photoluminescence measurements are performed in a temperature controlled liquid helium cryostat with internal $x-y$ nanopositioners, essential for stability and relocating a given nanocavity. The pump beam is focused by a $0.5 \mathrm{NA}$ reflecting microscope objective onto the sample, resulting in a spot size of $1 \mu \mathrm{m}$. The emission from the sample is collected by the same microscope objective, analyzed with a spectrometer, and detected by an InGaAs CCD array. In this geometry we are utilizing the leak of the cavity mode out the top to observe photoluminescence from a quantum dot coupled to it.

We are varying several parameters: the nanocavity $Q$; where the nanocavity mode peak is spectrally relative to the distribution of the photoluminescence from the ground state of the dots; whether or not the nanocavity exhibits strong coupling with $\mathrm{cw}$ low-power nonresonant excitation; ps versus gated $\mathrm{cw} 770 \mathrm{~nm}$ excitation. We expect to see lasing due to gain from a few quantum dots, similar to the room temperature lasing reported using $\sim 80$ dots in photonic crystal cavities with $\mathrm{Q} \sim 2000$ [5,2]. But here there is a single layer of dots of density $300-400 / \mu \mathrm{m}^{2}$, compared with their 5 layers of the same density; the mode volumes are roughly the same, but their $Q$ was 2000 compared to the $\sim 20,000$ here. Consequently, within the cavity linewidth, one expects the number of dots contributing to lasing here would be down by $(1 / 5)(2000 / 20000)=0.02$. That factor would reduce their 80 quantum dots to 1.6 here. We will study the "softness" of the threshold; the change in slope is expected to be smaller the higher the $Q$ and the larger the fraction of spontaneous emission that goes into the lasing mode (effective $\beta$ ) $[5,2]$. An interesting open question is the possibility of seeing lasing with a single quantum dot, as predicted in Fig. 9 of [2] provided $\gamma<1 \mathrm{meV}$ and $\mathrm{Q}>10,000$.

1. Y. Akahane, T. Asano, B.-S. Song,and S. Noda, "High- $Q$ photonic nanocavity in a two-dimensional photonic crystal." Nature 425, 944-947 (2003).

2. T. Yoshie, O. B. Shchekin, H. Chen, D. G. Deppe, and A. Scherer, "Planar photonic crystal nanolasers (II): Low-threshold quantum dot lasers," IEICE Trans. Electron. E87-C, 300-307 (2004).

3. T. Yoshie, A. Scherer, J. Hendrickson, G. Khitrova, H. M. Gibbs, G. Rupper, C. Ell, O. B. Shchekin, and D. G. Deppe, "Vacuum Rabi splitting with a single quantum dot in a photonic crystal nanocavity, " Nature 432, 200-203 (2004).

4. J. P. Reithmaier, G. Sęk, A. Löffler, C. Hoffmann, S. Kuhn, S. Reitzenstein, L. V. Keldysh, V. D. Kulakovskii, T. L. Reinecke, and A. Forchel, "Strong coupling in a single quantum dot-semiconductor microcavity system," Nature 432, 197-200 (2004)

5. T. Yoshie, O. B. Shchekin, H. Chen, D. G. Deppe, and A. Scherer, "Quantum dot photonic crystal lasers," Electron. Lett. 38, 967-968 (2002). 